\title{
Predictors of response to treatment for chronic
}

\section{fatigue syndrome}

\author{
RICHARD P. BENTALL, PAULINE POWELL, FRED J. NYE \\ and RICHARD H. T. EDWARDS
}

\begin{abstract}
Background Controlled trials have shown that psychological interventions designed to encourage graded exercise can facilitate recovery from chronic fatigue syndrome.
\end{abstract}

\begin{abstract}
Aims To identify predictors of response to psychological treatment for chronic fatigue syndrome.
\end{abstract}

\begin{abstract}
Method Of II4 patients assigned to equally effective treatment conditions in a randomised, controlled trial, 95 completed follow-up assessments. Relationships between variables measured prior to randomisation and changes in physical functioning and subjective handicap at I year were evaluated by multiple regression.
\end{abstract}

Results Poor outcome was predicted by membership of a self-help group, being in receipt of sickness benefit at the start of treatment, and dysphoria as measured by the Hospital Anxiety and Depression scale. Severity of symptoms and duration of illness were not predictors of response.

\section{Conclusions Poor outcome in the psychological treatment of chronic fatigue syndrome is predicted by variables that indicate resistance to accepting the therapeutic rationale, poor motivation to treatment adherence or secondary gains from illness.}

Declaration of interest None. Financial support from the Linbury Trust.
Chronic fatigue syndrome (CFS) is associated with muscular and cardiovascular deconditioning (De Lorenzo et al, 1998), circadian desynchrony (Williams et al, 1996), sleep abnormalities (Morriss et al, 1997) and cortisol deficiency (Cleare et al, 1995). It has been proposed that these signs are consequences of disrupted cycles of activity, and that CFS is maintained by illness beliefs that encourage activity avoidance (Wessely et al, 1998). Psychological interventions that encourage exercise are effective treatments for CFS (Sharpe et al, 1996; Deale et al, 1997; Powell et al, 2001). We tested the hypotheses that variables indicative of rigid illness beliefs (duration of untreated illness, membership of a support group advocating exercise avoidance, belief in physical illness), limited emotional resources (concurrent dysphoria, receipt of antidepressant medication, sleep problems), poor social support (living alone or as a lone parent), or probable loss of benefits on recovery, would predict poor response to this kind of treatment.

\section{METHOD}

\section{Participants}

Participants were 114 patients recruited from a chronic fatigue clinic and an infectious diseases out-patient clinic and assigned to three treatment groups in a randomised, controlled trial of a brief psychological intervention of chronic fatigue syndrome (Powell et al, 2001). Thirty-four patients assigned to a no-treatment control group were not included in this study, and a further 36 patients eligible for the trial did not take part -16 because they were unable to attend, 8 because they were in receipt of other therapy and 12 because they refused. All participants fulfilled the Oxford criteria for CFS (Sharpe et al, 1991) and scored less than 25 on the physical functioning sub-scale of the 36-item short form health survey (SF36; Ware \&
Sherbourne, 1992). This sub-scale has a scoring range of 10 to 30 , where 10 indicates maximum physical limitation in selfcare and 30 indicates the ability to do vigorous sports. The following exclusion criteria were employed:

(a) further physical investigations or other treatments including antidepressant therapy (unless the same dose had been taken for at least 3 months without improvement);

(b) psychotic illness, somatisation disorder, eating disorder or a history of substance misuse;

(c) confinement to wheelchair or bed.

\section{Treatment methods}

The trial compared three different 'dosages' (defined in terms of therapist time and follow-up telephone contacts with the therapist) of an educational intervention in which patients were taught evidencebased physiological explanations of their symptoms and were encouraged to undertake a home-based graded exercise programme. The 'minimum intervention' patients received two individual treatment sessions and two telephone follow-up calls, plus access to a telephone helpline. The 'telephone intervention' patients received an additional seven planned follow-up calls, and the 'maximum intervention' patients received an additional seven face-to-face sessions over 4 months.

\section{Assessments}

Clinical and demographic data collected from the patients prior to recruitment to the trial included duration of illness, gender, receipt of antidepressant therapy and sickness benefits, membership of a self-help group (all assessed as yes/no variables) and belief in a physical cause of CFS assessed by means of a four-point scale ('definitely physical', 'mainly physical', 'equally physical and psychological/stress-related' and 'mainly psychological/stress-related') derived from a measure of CFS illness beliefs developed by Wood et al (1991). Self-rated validated outcome measures sent by post were assessed before randomisation, and 3, 6 and 12 months after the beginning of treatment. Primary outcomes were measured on the physical functioning sub-scale of the SF36, and on the fatigue scale (Chalder et al, 1993: range 0-11, scores greater than 3 indicate excessive fatigue). At baseline the mean score for physical 
functioning of the treated patients was 16.0 and their mean score on the fatigue scale was 10.28. Secondary outcome measures assessed before treatment and at follow-up included the London Handicap Scale (Harwood et al, 1994), a multi-dimensional assessment of mobility, physical independence, occupational functioning, social integration and economic self-sufficiency which yields a global handicap score; the Hospital Anxiety and Depression (HAD) scale (Zigmond \& Snaith, 1983; scores above 10 indicate caseness); and a four-item sleep problem questionnaire (Jenkins et al, 1988).

\section{Statistical analysis}

Outcome data were available for 95 of the treated patients, representing loss from the study of $17 \%$ of the sample. For those who dropped out, the last recorded data point was carried forward prior to analysis. We analysed predictors of improvement by multiple regression, entering all variables at once, a method which is more conservative and less prone to type 1 errors than the more commonly used stepwise method. As multiple regression allows one variable to be included for every 10 participants, we had sufficient power to include nine predictors (Kleinbaum et al, 1988). Although treatment dose bore no relation to outcome in our main outcome analysis at 1 year (Powell et al, 2001) we included it as a predictor in all analyses by coding dose as 1 (minimum treatment), 2 (telephone treatment) and 3 (maximum treatment). Initial scores on the dependent variables were included in the regression equations to control for systematic relationships between initial scores and outcome.

Predictors were chosen on the basis of the hypotheses listed in the introduction. We therefore included the following binary variables: membership of a self-help group, living alone or as a lone parent, being in receipt of sickness benefits, and antidepressant therapy. Continuous variables included in the analysis were duration of illness, illness beliefs, and sleep disturbance scores. Depression and anxiety scores from the HAD scales were also included, but because these were highly correlated (Pearson $r=0.56, P<0.001$, one-tailed), they were summed into a single dysphoria variable to avoid the problem of multi-collinearity. Initial scores on these variables are shown in Tables 1 and 2. The mean physical functioning score of the members of the selfhelp groups was 14.29 compared with
Table I Initial scores on predictor variables: binary variables (total $n=95$ )

\begin{tabular}{lll}
\hline Predictor variable & $n$ & (\%) \\
\hline Membership of self-help group & 17 & 18 \\
Living alone or lone parent & 19 & 20 \\
In receipt of antidepressant therapy & 14 & 15 \\
In receipt of sickness benefit & 37 & 39 \\
\hline
\end{tabular}

16.38 for non-members, indicating that members were more impaired at the outset $(t=2.80, P<0.01,112$ d.f. $)$.

\section{RESULTS}

The outcome of these interventions at 1year follow-up has been presented elsewhere (Powell et al, 2001). Patients in the three treatment groups showed significantly greater improvement on all primary and secondary outcome variables in comparison with patients in the control group. However, no significant difference was found between the outcomes associated with the three treatment dosages. Only two of the 34 patients in the control group met our predetermined criteria for a clinically significant improvement, which was a final score of 25 or more or an increase of 10 or more on the physical functioning scale (virtually equivalent to normal functioning). However, 26 of 37 patients in the 'minimum intervention' group, 27 of 39 in the 'telephone intervention' group and 26 of 38 in the 'maximum intervention' group improved.

\section{Predictors of change in physical functioning scores}

Our main outcome measure in the trial was change on the SF36 physical functioning scale, which was approximately normally distributed, with an average improvement of 8.83 points (s.d.=5.23, skewness $-0.557)$, indicating that most of the patients who were followed-up improved. When this was used as the dependent variable in the regression model, the equation was highly significant $\left(r^{2}=0.38, F=6.29\right.$, $P<0.001)$. Standardised regression coefficients and significance tests for each of the predictors are given in Table 3. Apart from initial physical functioning scores, poor response to treatment was predicted by (in order of predictive power) dysphoria scores, membership of a self-help group, and receipt of sickness benefit. Living arrangements (alone or as a single parent), receipt of antidepressant therapy, sleep disturbance, duration of illness, dose of treatment and-surprisingly - initial illness beliefs did not add significantly to the model.

\section{Predictors of change in London Handicap Scale scores}

Fatigue scores (both change and final), our second primary outcome measure, deviated severely from the normal distribution and were unsuited to regression analysis. Therefore, to assess the validity of the above findings, the analyses were repeated using change scores on the London Handicap Scale, which were normally distributed. The regression equation was again highly significant $\left(r^{2}=0.38, F=6.13, P<0.001\right)$. In addition to initial scores on the scale, predictors of poor response on this scale were membership of a self-help group, receipt of sickness benefit, belief in a physical cause of illness, and dysphoria (Table 3).

\section{Analyses excluding initial scores}

It is difficult to interpret the theoretical significance of the observed relationships between initial and change scores as these may simply reflect the fact that those who were most ill had most opportunity to change. The analyses were therefore repeated with final scores as the dependent variables. These were less than ideal for analysis because they had highly non-normal distributions with scores highly

Table 2 Initial scores on predictor variables: continuous variables

\begin{tabular}{lrr}
\hline Predictor variable & Mean & s.d. \\
\hline HAD depression score (range 0-2I, > I0 indicates depression) & 8.82 & 3.72 \\
HAD anxiety score (range 0-2I, > I0 indicates anxiety) & 20.28 & 4.63 \\
Sleep score (range 0-20, where 20 indicates maximum disturbance) & 12.83 & 4.76 \\
Cause of illness (range I-4, where I indicates definitely physical and 4 indicates mainly y & 2.15 & 0.84 \\
$\quad$ psychological) & \\
\hline
\end{tabular}

HAD, Hospital Anxiety and Depression scale. 
Table 3 Standardised regression coefficients, $t$ and significance values for predictor variables in regression equations predicting changes in SF36 physical functioning scores and London Handicap Scale scores at I-year follow-up

\begin{tabular}{|c|c|c|c|}
\hline Dependent variable and predictors & $\beta$ & $t$ & $P$ \\
\hline \multicolumn{4}{|l|}{ Change in physical functioning (SF36) } \\
\hline Membership of support group & -0.28 & -3.45 & 0.001 \\
\hline Living alone or as lone parent & 0.12 & 1.52 & 0.13 \\
\hline Receipt of sickness benefit & -0.21 & -2.57 & 0.01 \\
\hline Duration of illness & 0.04 & 0.42 & 0.68 \\
\hline Receipt of antidepressant medication & 0.11 & 1.36 & 0.18 \\
\hline Dysphoria (HAD) & -0.34 & -3.75 & 0.001 \\
\hline Belief in physical cause & -0.08 & -0.88 & 0.38 \\
\hline Sleep problems & 0.12 & I.31 & 0.38 \\
\hline Treatment dose & -0.06 & -0.70 & 0.49 \\
\hline Initial physical functioning & -0.53 & -5.84 & 0.001 \\
\hline \multicolumn{4}{|l|}{ Change in London Handicap Scale score } \\
\hline Membership of support group & -0.30 & -3.79 & 0.001 \\
\hline Living alone or as lone parent & 0.13 & 1.66 & 0.10 \\
\hline Receipt of sickness benefit & -0.22 & -2.45 & 0.02 \\
\hline Duration of illness & -0.04 & -0.47 & 0.64 \\
\hline Receipt of antidepressant medication & 0.11 & 1.41 & 0.16 \\
\hline Dysphoria (HAD) & -0.20 & -2.13 & 0.04 \\
\hline Belief in physical cause & -0.19 & -2.28 & 0.03 \\
\hline Sleep problems & -0.08 & -0.86 & 0.39 \\
\hline Treatment dose & -0.04 & -0.53 & 0.60 \\
\hline Initial handicap score & -0.60 & -5.86 & 0.001 \\
\hline
\end{tabular}

HAD, Hospital Anxiety and Depression scale; SF36, 36-item short-form health survey.

skewed towards maximum values (physical functioning mean 24.74, skewness -0.96 ; London Handicap Scale mean 76.27, skewness -0.22$)$. None the less, the regression equation for final physical functioning was significant $\left(r^{2}=0.34, F=5.16, P<0.001\right)$, the same three predictors were retained in the equation, but initial physical functioning scores no longer predicted outcome. Similarly, for final London Handicap Scale scores, a significant regression equation was calculated $\left(r^{2}=0.39, \quad F=6.59\right.$, $P<0.001$ ), with the same four predictors retained, and no significant contribution to the model from initial London Handicap Scale scores.

\section{Supplementary analyses}

In the above analyses we assumed that the scores of those who dropped out had not changed from their last recorded data points. To test the impact of this assumption, we repeated the main analyses assuming that all of those who dropped out had done poorly by assigning them the relevant mean scores of the untreated control group. For changes in physical functioning, dysphoria, membership of a support group and receipt of state benefits remained predictors. For changes in the London Handicap Scale scores, dysphoria, membership of a support group, receipt of benefits, and physical illness attributions were all retained. As a further check for the validity of our assumptions, we repeated the analyses with all data from those who dropped out excluded (leaving a sample size of 95). In this case, dysphoria and membership of a support group remained predictors of changes in physical functioning, but receipt of benefits was no longer a predictor. However, for changes in scores on the London Handicap Scale, only membership of a support group remained as a predictor.

\section{DISCUSSION}

\section{Variables that did not predict treatment response}

Variables measured on admission to the trial predicted a sizeable proportion of the variance in outcomes - nearly $40 \%$ in most of the analyses. Variables that failed to predict response to treatment were, in many ways, as interesting as those that did. Our supplementary analyses of raw outcome scores indicated that severity of initial scores on these measures did not predict improvement. Duration of illness, receipt of antidepressant therapy, living circumstances and (as we expected from our main trial data) treatment dose also failed to predict outcome. The implication of these negative findings is that severity and chronicity of core CFS symptoms are not impediments to clinical response, other things being equal, and that psychological treatment should therefore be offered to patients whose symptoms are fairly severe. Although non-ambulatory patients were not included in this study, we have elsewhere reported the successful treatment of wheelchair-bound patients using an extended version of our treatment programme (Powell et al, 1999).

\section{Variables predicting treatment response}

The most robust predictors of poor treatment response were membership of a support group and concurrent emotional difficulties. Although we found that support group members were more ill than non-members on admission to the study, this was controlled for in our regression analyses, and therefore cannot explain the impact that membership had on outcomes. Receipt of benefits and belief in exclusively physical causes of CFS were also predictors of poor response in some of the analyses. These variables may be indicators of poor motivation for treatment adherence. Individuals who are emotionally distressed, who will experience financial difficulties when improving, and who are initially sceptical about the value of psychological approaches, may have difficulty persisting with a graded exercise programme.

\section{Consistency with previous findings}

Consistent with our findings, Sharpe et al (1992) followed-up people with CFS attending an infectious disease clinic and found that poor outcome was associated with emotional disorder, belief in a viral illness and membership of a self-help organisation (other predictors of poor response were limiting exercise, avoiding alcohol and changing employment). In a 4-year follow-up of patients taking part in an open, uncontrolled trial of psychological treatment for CFS, Bonner et al (1994) found that poor response was predicted 
by emotional disorder and that there was also a trend for physical illness beliefs to predict a poor response; however, no relationship was found between response and membership of a support group. The current finding that receipt of sickness benefit at the start of treatment is associated with poor outcome is consistent with a report by Barsky \& Borus (1999) that disability payments are associated with worse symptomatic outcome after medical treatment.

Mechanic (1986) reported that interest groups who adhere to particular theories of illness may play a part in reinforcing illness beliefs. In the presence of such a group, a personal problem becomes a shared social problem. Although there are undoubted psychological benefits from this kind of support (McCully et al, 1996), there have been reports that the advice dispensed by some CFS groups may have a negative effect on recovery, for example by advocating the avoidance of activity (Abbey, 1993; Surawy et al, 1995). It may be counterproductive to discourage patients from joining such groups, however; instead, clinicians and researchers should forge constructive alliances with support groups, to ensure that patients receive advice that is consistent and evidence-based. In this context, we should like to acknowledge that our work has been assisted by support groups in the Merseyside area, who have had an active role in ensuring that our treatment programme is now being offered as a routine National Health Service therapy.

\section{Limitations of this study}

The randomised clinical trial from which these data were taken had several limitations, which should be noted. The Oxford criteria for CFS, used as an entry criterion, are broader than the now widely used Centers for Disease Control definition (Fukuda, 1994). More importantly, outcome was measured by self-report. Although this had the advantage of minimising the possibility of researcher bias in assessing outcome, it would have been better to have included an objective measure of exercise capacity. None the less, it seems implausible that these limitations can account for the results discussed here. In general, the observation that an intervention designed to affect illness beliefs had positive effects, coupled with the observation that variables indicative of poor treatment motivation predicted poor outcome, is consistent with

\section{CLINICAL IMPLICATIONS}

- The severity and chronicity of chronic fatigue syndrome symptoms do not predict response to psychological treatment aimed at encouraging graded exercise. This kind of treatment should therefore be available to all patients.

- Treatment response is predicted by concurrent emotional difficulties and receipt of state benefits, factors that may undermine motivation to persist with treatment.

- Membership of a support group is also associated with poor treatment response, probably because such groups sometimes advise against graded exercise interventions. This observation highlights the importance of building a constructive alliance between services and support groups.

\section{LIMITATIONS}

- A broad definition of chronic fatigue syndrome was used as an entry criterion.

- Seventeen per cent of patients were lost to follow-up.

- Only subjective measures of outcome were recorded; there was no objective assessment of exercise capacity.

RICHARD P. BENTALL, PhD, University of Manchester; PAULINE POWELL, PhD, FRED J. NYE, FRCP, Roya Liverpool Broadgreen Trust; RICHARD H. T. EDWARDS, FRCP (retired), formerly University of Liverpool, UK

Correspondence: Professor Richard Bentall, Department of Psychology, University of Manchester, Coupland I Building, Oxford Road, Manchester MI3 9PL, UK

(First received I August 200I, final revision 10 April 2002, accepted 17 April 2002)

our overall hypothesis that attitudinal factors play a part in maintaining CFS symptoms.

\section{ACKNOWLEDGEMENT}

This research was supported by a grant from the Linbury Trust.

\section{REFERENCES}

Abbey, S. (1993) Somatization, illness attribution and the sociocultural psychiatry of chronic fatigue syndrome. In Chronic Fatigue Syndrome (ed. S. Straus), pp. 238-261. Chichester: John Wiley \& Sons.

Barsky, A. \& Borus, J. (1999) Functional somatic symptoms. Annals of Internal Medicine, 130, 910-92

Bonner, D., Ron, M., Chalder, T., et al (1994) Chronic fatigue syndrome: a follow up study. Journal of Neurology, Neurosurgery and Psychiatry, 57, 617-621.

Chalder, T., Berelowitz, G., Pawlikowska, T., et al (1993) Development of a fatigue scale. Journal of Psychosomatic Research, 37, 147-153.

Cleare, A., Bearn, J., Allain, T., et al (1995)

Contrasting neuroendocrine responses in depression and chronic fatigue syndrome. Journal of Affective Disorders, 34, 283-289.
Deale, A., Chalder, T., Marks, I., et al (1997) Cognitive behavioural therapy for chronic fatigue syndrome: a randomised controlled trial. American Journal of Psychiatry, I54, 408-4I4.

De Lorenzo, F., Xiao, H., Mukherjee, M., et al (1998) Chronic fatigue syndrome: physical and cardiovascular deconditioning. Quarterly journal of Medicine, 91, 475-48I.

Fukuda, K. (1994) The chronic fatigue syndrome: a comprehensive approach towards its definition and study. Annals of Internal Medicine, I2I, 953-959.

Harwood, R., Rogers, A., Dickinson, E. et al (1994) Measuring handicap: methods, and a model. Quality in Health Care, 3, 53-57.

Jenkins, D., Stanton, B., Niemcryk, S., et al (1988) A scale for the estimation of sleep problems in clinical research. Journal of Clinical Epidemiology, 4I, 313-32I.

Kleinbaum, D. G., Kupper, L. L. \& Muller, K. E. (1988) Applied Regression Analysis and Other Multivariable Methods (2nd edn). Boston, MA: PWS-Kent.

McCully, K., Sisto, S. \& Natelson, B. (1996) Use of exercise for treatment of chronic fatigue syndrome. Sports Medicine, I, 35-48.

Mechanic, D. (1986) The concept of illness behaviour: culture, situation and personal predisposition. Psychological Medicine, 16, I-7.

Morriss, R., Wearden, A. \& Battersby, L. (1997) The relation of sleep difficulties to fatigue, mood and 
disability in chronic fatigue syndrome. Journal of Psychosomatic Research, 42, 597-605.

Powell, P., Edwards, R. \& Bentall, R. (1999) The treatment of wheelchair-bound chronic fatigue syndrome patients: two case studies of a pragmatic treatment approach. Behavioural and Cognitive Psychotherapy, 27, 249-260.

_ , Bentall, R., Nye, F., et al (200I) Randomised controlled trial of patient education to encourage graded exercise in chronic fatigue syndrome. BMJ, $\mathbf{3 2 2}$, 387-390.

Sharpe, M., Archard, L., Banarvala, J., et al (199I) A report - chronic fatigue syndrome: guidelines for research. Journal of the Royal Society of Medicine, $\mathbf{8 4}$ |18-121.
_ , Hawton, K., Seagrott, V., et al (1992) Follow up of patients presenting with fatigue to an infectious diseases clinic. BMJ, 305, 147-152.

_, _, Simkin, S., et al (1996) Cognitive behaviour therapy for the chronic fatigue syndrome: a randomised controlled trial. BMJ, 32I, 22-26.

Surawy, C., Hackmann, A., Hawton, K., et al (1995)

Chronic fatigue syndrome: a cognitive approach.

Behavioural Research Therapy, 33, 535-544.

Ware, J. \& Sherbourne, C. (1992) The MOS 36-item short form health survey (SF36). Medical Care, 30 473-483.
Wessely, S., Hotopf, M. \& Sharpe, M. (1998) Chronic Fatigue and Its Syndromes. Oxford: Oxford University Press.

Williams, G., Piromohamed, J., Minors, D., et al (1996) Dissociation of body-temperature and melatonin secretion circadian rhythms in patients with chronic fatigue syndrome. Clinical Physiology, 16, 327-337.

Wood, G., Bentall, R., Gopfert, M., et al (199I) A comparative psychiatric assessment of patients with chronic fatigue syndrome and muscle disease. Psychological Medicine, 2I, 619-628.

Zigmond, A. \& Snaith, R. (1983) The Hospital Anxiety and Depression Scale. Acta Psychiatrica Scandinavica, 67, 361-370. 